\title{
An improved CE/SE scheme for incompressible multiphase flows and its applications
}

\section{Duoxing Yang*}

Research Institute of Innovative Technology for the Earth, 9-2, Kizugawadai, Kizugawa-Shi, Kyoto, 619-0292, Japan and

Key Laboratory of Crustal Dynamics, Institute of Crustal Dynamics, Chinese Earthquake Administration, P.O. Box 2855, Beijing, 100085, China Fax:+81-774-752312

E-mail: dxyangcea@yahoo.com

*Corresponding author

\section{Deliang Zhang}

Institute of Mechanics, Chinese Academy of Sciences,

No. 15 Beisihuanxi Road, Beijing, 100190, China

E-mail: dlzhang@imech.ac.cn

\begin{abstract}
A new space-time conservation element-solution element (CE/SE) scheme, which is based on the hexahedron mesh, is proposed by constructing a new structure of solution elements (SEs) and conservation elements (CEs) and extended to two-dimensional incompressible multiphase flows. This scheme is applied to several incompressible flow problems, such as lid-driven cavity flow, dam break flow, motions of falling droplets, flow instability and water-kerosene interaction. The numerical results and their comparison with lecture results and experimental data are presented. The comparisons demonstrate that the computational scheme developed currently is clear in physical concept, easy to be implemented and highly accurate and efficient for the above-mentioned problems. The highlight is that the pretreatment method is proposed, transforming the elliptic-parabolic equations to the hyperbolic equations, which can be directly simulated by the improved CE/SE scheme. Thus, the improved $\mathrm{CE} / \mathrm{SE}$ scheme can be applied to incompressible multiphase flows widely.
\end{abstract}

Keywords: CE/SE scheme; multiphase flows; computational fluid dynamics; CFD.

Reference to this paper should be made as follows: Yang, D. and Zhang, D. (2013) 'An improved CE/SE scheme for incompressible multiphase flows and its applications', Progress in Computational Fluid Dynamics, Vol. 13, No. 5, pp.285-295.

Biographical notes: Duoxing Yang received his $\mathrm{PhD}$ in Geology Engineering from the Institute of Geology and Geophysics, Chinese Academy of Sciences. He is a Visiting Researcher of the Research Institute of Innovative Technology for The Earth, Japan. Since 2008, he is an Associate Professor in the Institute of Crustal Dynamics, CEA, conducting researches on heat and mass transfer in porous media, multiphase flow simulation and $\mathrm{CO}_{2}$ geological storage.

Deliang Zhang is a Professor in the Institute of Mechanics, Chinese Academy of Sciences, working on computational fluid dynamics. 


\section{Introduction}

The incompressible multiphase flows are highly non-linear phenomena. Numerical simulations have become popular and are playing more and more important roles in the incompressible multiphase flow research. However, two main challenges are still confronted in the numerical methods: the numerical scheme for the governing equations and the special treatments for the fluid-fluid interfaces. Recently, the numerical research of incompressible multiphase flow is mainly focusing on numerical schemes for Navier-Stokes equations (NSE) and a method for interface treatment. Method research on NSE solver includes Lattice Boltzmann equation (LBE) method (Nourgaliev et al., 2003), pseudocompressibility method (Nourgaliev et al., 2004), projection method (Ni et al., 2006) and non-linear multigrid method (Shin et al., 2011), etc. Methods for interface treatment involve level-set method (Osher and Fedkiw, 2001), volume of fluid, ghost fluid method, a coupling method of volume-of-fluid and level set method (CLSVOF), particle level-set method and local front reconstruction method (LFRM) (Shin et al., 2011), etc. The hybrid particle level set (HPLS) method based on massless marker particles was proposed by Enright et al. (2002), which combines the merits of the Lagrangian approach and the Eulerian level set method. The HPLS is a smart technique for improved mass conservation in a fluid flow and is effective for tracing fluid-fluid interfaces.

The space-time conservation element-solution element (CE/SE) method, first proposed by Chang (1995), is a novel computational fluid dynamics (CFD) method for conservation laws. This method is distinctive from traditional numerical method and is based on the global and local flux conservation in a space-time domain by introducing conservation elements (CEs) and solution elements (SEs). The CE/SE method unifies space and time and treats them as a single entity, preserving global and local flux conservation in both space and time. Typically, both independent flow variables and their derivatives are treated as unknowns and are solved for simultaneously. The $\mathrm{CE} / \mathrm{SE}$ method has been used for accurate numerical solutions involving shocks (Chang, 1995), viscous flows (Guo et al., 2004), magneto-hydro-dynamic (MHD) flows (Zhang et al., 2006), multi-material elastic-plastic flows (Wang et al., 2009) and dilute gas-particle flows (Wang et al., 2011), etc. Recently, Zhang et al. (2002) proposed the $\mathrm{CE} / \mathrm{SE}$ scheme by using quadrilateral and hexahedral meshes. Wang et al. (2009) proposed two-dimensional high-accuracy CE/SE schemes, which is based on the rectangular mesh. However, the CE/SE schemes above-mentioned have been proposed for compressible flow problems, which are governed by hyperbolic equations.
The features described above make the CE/SE method a good candidate for problems such as Marangoni effects (Savino et al., 2003), microconfined shear flow (Sibillo et al., 2006), viscoelastic fluid (Harvie et al., 2008), etc. However, before testing the $\mathrm{CE} / \mathrm{SE}$ method on these demanding tasks, one has to first be able to apply the CE/SE method to incompressible multiphase flow problems (governed by elliptic-parabolic equations) on a routine basis.

The aim of the present work is to construct a simple and accurate two-dimensional CE/SE scheme for incompressible multiphase flows. We propose a new definition of SE and $\mathrm{CE}$ and construct an improved $\mathrm{CE} / \mathrm{SE}$ scheme based on the hexahedron mesh. Furthermore, we apply the improved $\mathrm{CE} / \mathrm{SE}$ scheme to solve the problems of two-dimensional incompressible multiphase flows. As to the knowledge of the authors, it is the first time to use the CE/SE method to solve these problems. The HPLS technique is used for tracing fluid-fluid interfaces. For verifying the accuracy, resolution, and efficiency of the improved $\mathrm{CE} / \mathrm{SE}$ scheme, several validation cases, including cavity flow, dam break, falling droplets, flow instability and water-kerosene interaction are numerically simulated. The computational results are also compared with the results from experiments and other literatures.

\section{Governing equations}

Based on the continuum surface force CSF) mode, a set of governing equations for mass and momentum of incompressible multiphase flows is obtained as follows (Ni et al., 2006):

Continuity equation:

$$
\frac{\partial u_{i}}{\partial x_{i}}=0
$$

Momentum equation:

$$
\begin{aligned}
\frac{\partial u_{i}}{\partial t}+\frac{\partial}{\partial x_{j}}\left(u_{i} u_{j}\right)= & -\frac{1}{\bar{\rho}} \frac{\partial P}{\partial x_{i}}+\frac{1}{\operatorname{Re} \bar{\rho}} \frac{\partial}{\partial x_{j}}\left(\bar{\mu}\left(\frac{\partial u_{i}}{\partial x_{j}}+\frac{\partial u_{j}}{\partial x_{i}}\right)\right) \\
& -\frac{1}{\bar{\rho} W e} \vec{F}_{S V}-\frac{g_{i}}{F r}
\end{aligned}
$$

where $u_{i}, u_{j}$ and $P$ are the dimensionless velocities and pressure, respectively. The Reynolds, Froude and Weber numbers are denoted as $\operatorname{Re}=\frac{\rho_{o} L U}{\mu_{o}}, F r=\frac{U^{2}}{g L}$, and $W e=\frac{\rho_{o} L U^{2}}{\sigma_{o}}$ respectively. $\bar{\rho}, \bar{\mu}$ are the dimensionless ratios of the viscosity and density. For simplicity, $\lambda_{\rho}$ and $\lambda_{\mu}$ are presented as $\bar{\rho}$ and $\bar{\mu}$ respectively. The surface tension force for the level set approach is reformulated as a volume force (Brackbill et al., 1992), 


$$
\vec{F}_{S V}=k(\varphi) \delta_{\alpha}(\varphi) \nabla \varphi
$$

where $\varphi$ is a smooth level set function, which is positive outside the interface, negative inside the interface and zero at the interface. $\delta$ is a surface tension delta function and $\kappa$ is the interface front curvature. To prevent numerical instability, it is necessary to smooth the values of the density $\rho_{\varepsilon}$ and viscosity $\mu_{\varepsilon}$ as:

$$
\begin{aligned}
& \rho_{\varepsilon}(\varphi)=\lambda_{\rho}+\left(1-\lambda_{\rho}\right) H_{\varepsilon}(\varphi) \\
& \mu_{\varepsilon}(\varphi)=\lambda_{\mu}+\left(1-\lambda_{\mu}\right) H_{\varepsilon}(\varphi)
\end{aligned}
$$

where the Heaviside function (Enright et al., 2002) $H_{\varepsilon}(\varphi)$ is formulated as follows:

$$
H_{e}(\varphi)=\left\{\begin{array}{cl}
0 & \varphi<-\varepsilon \\
\frac{1}{2}\left(1+\frac{\varphi}{\varepsilon}+\frac{1}{\pi} \sin \left(\frac{\pi \varphi}{\varepsilon}\right)\right) & |\varphi| \leq \varepsilon \\
1 & \varphi>\varepsilon
\end{array}\right.
$$

\section{Interfaces tracing algorithm}

Based on level-set method (Osher and Fedkiw, 2001), the following equation will evolve the zero level set function:

$$
\frac{\partial \varphi}{\partial t}+u_{i} \frac{\partial \varphi}{\partial x_{i}}=0
$$

The above level set equation is solved using a five-order WENO discretisation and Runge-Kutta method. Sussman et al. (1994) presented a reinitialisation equation insure that values for level set function will not be greatly distorted. The reinitialisation equation can be reformulated as:

$$
\begin{aligned}
& \varphi_{\tau}+w \cdot \nabla \varphi=\operatorname{sign}_{\varepsilon}\left(\varphi_{o}\right) \\
& \varphi(\vec{x}, 0)=\varphi_{o}(\vec{x})
\end{aligned}
$$

where

$$
w=\operatorname{sign}_{\varepsilon}\left(\varphi_{o}\right) \nabla \varphi /|\nabla \varphi|,|\nabla \varphi|=\sqrt{\varphi_{x}^{2}+\varphi_{y}^{2}},
$$

and $\operatorname{sign}_{\varepsilon}\left(\varphi_{o}\right)=2\left(H_{\varepsilon}\left(\varphi_{o}\right)-1 / 2\right)$ the sign function. The numerical discretisation of the reinitialisation equation of the level set function will not preserve the total mass conservation. To overcome this difficulty, the HPLS method (Enright et al., 2002) can be presented to conduct the solution of the reinitialisation equation. HPLS method is a coupling method of Lagrangian method and Eulerian method, which merges the best aspects of Eulerian front capturing schemes and Lagrangian front-tracking methods for improved mass conservation in a fluid flow. Massless marker particles were inserted to correct mass loss in level set function by using the characteristic information of the escaped massless marker (as shown in Figure 1). The particle level set method maintains the nice geometric properties of level set method, and performs favourably in the conservation of mass and for interface resolution. The particle Lagrangian function is written as:

$$
\frac{d \bar{r}_{p}}{d t}=\vec{V}\left(\bar{r}_{p}\right)
$$

where $\bar{r}_{p}$ is the location of the particle, and $\bar{V}$ is the particle velocity. The third-order Runge-Kutta scheme is used to solve equation (8).

Figure 1 Schematic map of HPLS method

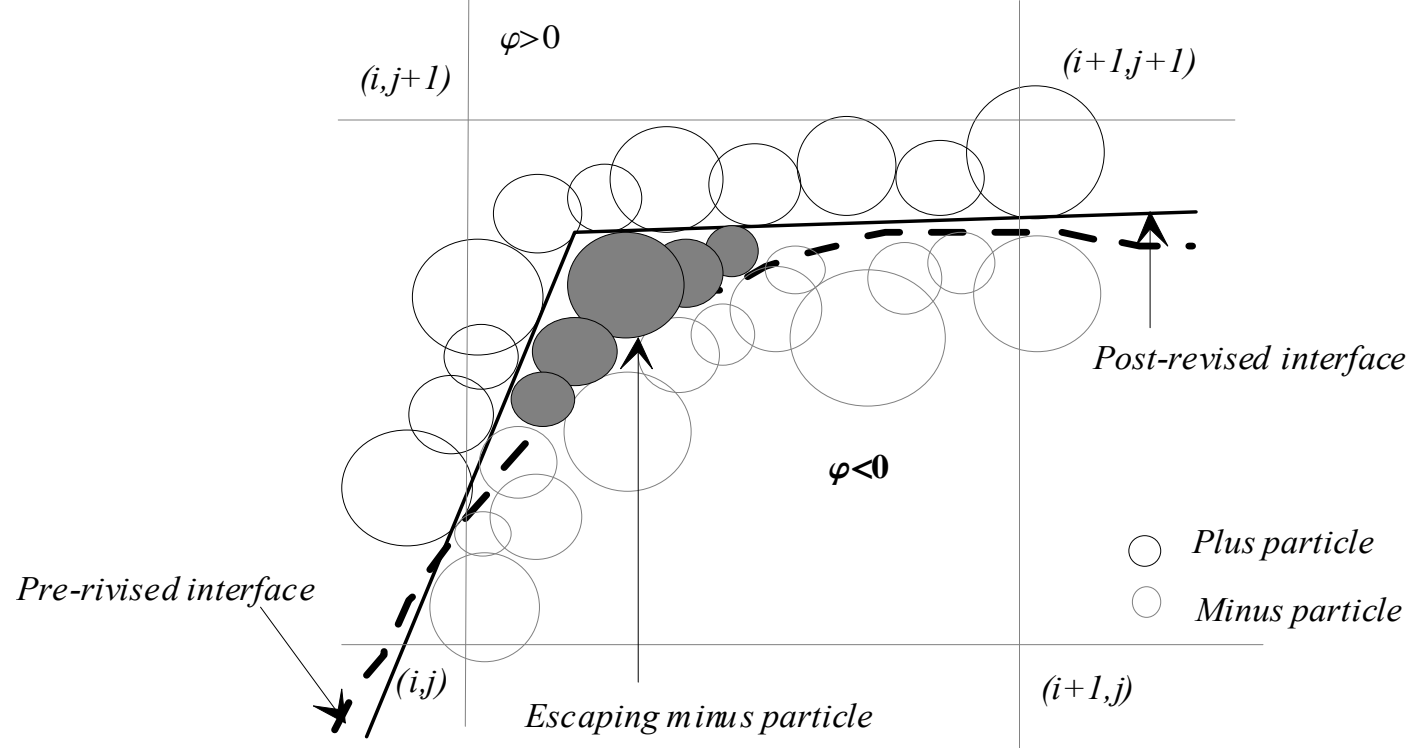


Figure 2 Mesh construction of the improved CE/SE method, (a) mesh points projection on $x y$ plane (b) $\mathrm{SE}\left(\mathrm{P}^{\prime}\right)$ (c) $\mathrm{CE}\left(\mathrm{P}^{\prime}\right)$

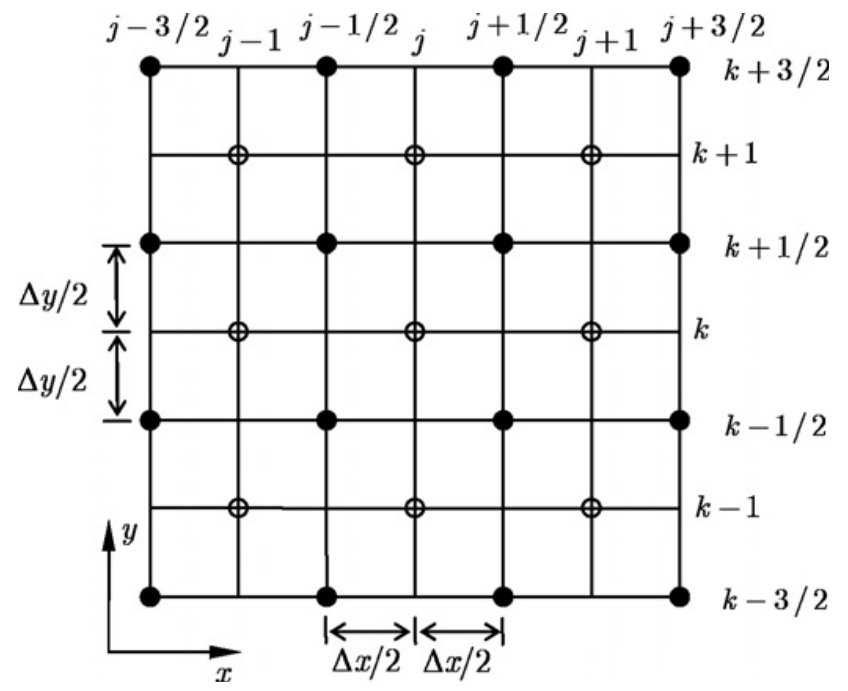

(a)

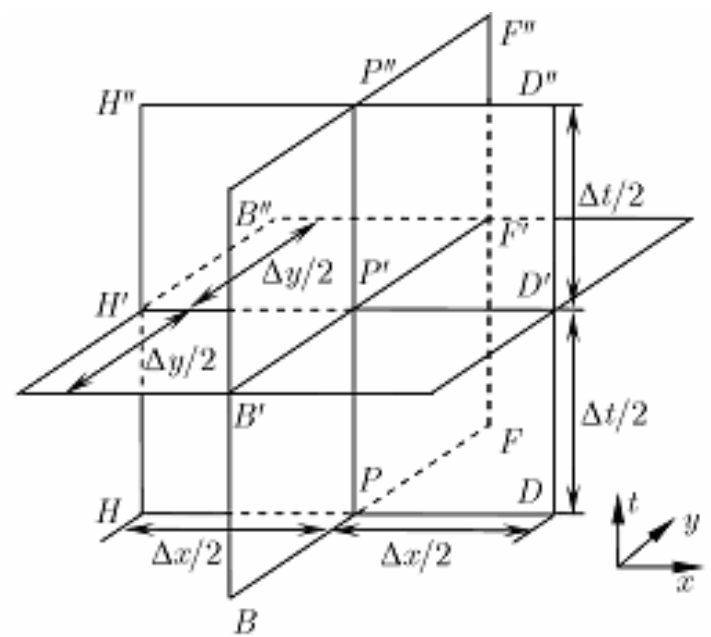

(b)

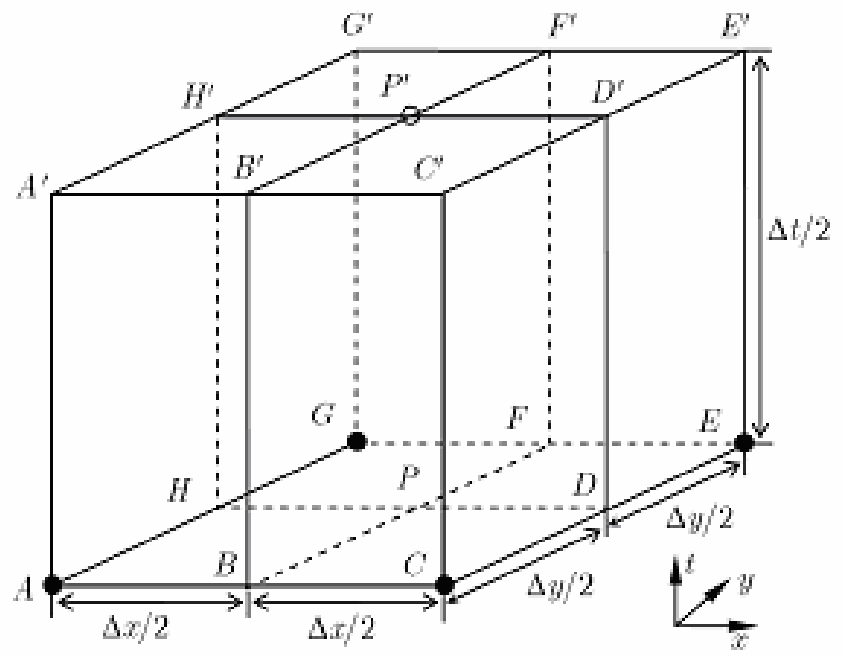

(c)

\section{Improved CE/SE method}

\subsection{Numerical scheme}

At first, the governing equations (1) and (2) can be rewritten in the form of conservation laws as

$$
\frac{\partial \mathbf{Q}}{\partial t}+\frac{\partial \mathbf{E}(\mathbf{Q})}{\partial x}+\frac{\partial \mathbf{F}(\mathbf{Q})}{\partial y}=\mathbf{S}(\mathbf{Q})
$$

with

$$
\begin{aligned}
& \mathbf{Q}=\left[\begin{array}{l}
0 \\
u \\
v
\end{array}\right], \quad \mathbf{E}=\left[\begin{array}{c}
u \\
u^{2}+\frac{P}{\bar{\rho}}-\frac{\tau_{x x}}{\bar{\rho} \operatorname{Re}} \\
u v-\frac{\tau_{y x}}{\bar{\rho} \operatorname{Re}}
\end{array}\right], \\
& \mathbf{F}=\left[\begin{array}{c}
v \\
u v-\frac{\tau_{x y}}{\bar{\rho} \operatorname{Re}} \\
v^{2}+\frac{P}{\bar{\rho}}-\frac{\tau_{y y}}{\bar{\rho} \operatorname{Re}}
\end{array}\right], \mathbf{S}=\left[\begin{array}{c}
0 \\
\frac{g_{x}}{F r}+\frac{\kappa(\varphi) \delta_{e}(\varphi)}{\bar{\rho} W e} \frac{\partial \varphi}{\partial x} \\
\frac{g_{y}}{F r}+\frac{\kappa(\varphi) \delta_{e}(\varphi)}{\bar{\rho} W e} \frac{\partial \varphi}{\partial y}
\end{array}\right]
\end{aligned}
$$

where $\mathbf{Q}, \mathbf{E}, \mathbf{F}, \mathbf{S}$ are vectors of primary variable, flux in $x$-direction, flux in $y$-direction and source, respectively. The normal and shear stress are given by

$$
\tau_{x x}=2 \mu \frac{\partial u}{\partial x}, \quad \tau_{y y}=2 \mu \frac{\partial v}{\partial y}, \quad \tau_{x y}=\tau_{y x}=\mu\left(\frac{\partial u}{\partial y}+\frac{\partial v}{\partial x}\right) .
$$

Here, a new type of SE and CE on the general hexahedron mesh is designed, which is different from that of Chang et al.'s (1999) original CE/SE method (see Figure 2). The construction of the new numerical scheme is simpler than Chang's and it is easy to be extended to three dimensional problems.

Let $(j, k, n)$ denote a set of space-time mesh points, where $n=0, \pm \frac{1}{2}, \pm 1, \pm \frac{3}{2} \ldots$ for time, $j=0, \pm \frac{1}{2}, \pm 1, \pm \frac{3}{2} \ldots$ for $x, k=0, \pm \frac{1}{2}, \pm 1, \pm \frac{3}{2} \ldots$ for $y$. A SE is defined as the vicinity of a mesh point and the whole space-time region is divided into non-overlapping $\mathrm{CE}$. Assume that the physical variables in every SE are approximated by the Taylor's expansions at the mesh point associated with the SE, and the conservation equation (9) is satisfied in every CE. Let $x_{1}=x, x_{2}=y, x_{3}=t$ be considered as the coordinates of a Euclidean space $E_{3}$. By means of the Gauss' divergence theorem, equation (9) is rewritten in form of

$$
\oint_{S(V)} \mathbf{H}_{m} \cdot d \mathbf{s}=\int_{V} S_{m} d V
$$


where $\mathbf{H}_{m}\left(E_{m}, F_{m}, Q_{m}\right)$ is the space-time flux vector, here $Q_{m}, E_{m}$ and $F_{m}$ are the components of vector $\mathbf{Q}, \mathbf{E}$ and $\mathbf{F}$, and respectively, and $S_{m}$ are the components of the source term vector. $S(V)$ is the boundary of an arbitrary space-time region $V$ in $E_{3}, d \mathbf{s}=d \sigma \cdot \mathbf{n}$ with $d \sigma$ and $\mathbf{n}$, respectively, being the area and the outward unit normal of a surface element on $S(V)$. Figure 2(a) shows the projection of mesh points on the $x-y$ plane, in which the interval between the mesh points $\bullet$ and $\circ$ is $\Delta t / 2$ in the time direction or $\frac{1}{2}$ in the mesh number $n$. For any point $P^{\prime}(j, k, n)$ on which the variables are solved, define the $\mathrm{SE}\left(\mathrm{P}^{\prime}\right)$ constituted by the three vertical planes intersecting at $P^{\prime}(j, k, n)$ and their neighbourhood space as demonstrated in Figure 2(b). Suppose that $Q_{m}, E_{m}$ and $F_{m}$ at point $(t, x, y)$ in SE $\left(P^{\prime}\right)$ are approximated by the second-order Taylor expansions at $P^{\prime}(j, k, n)$ i.e.,

$$
\begin{aligned}
& Q_{m}(d x, d y, d t)_{P^{\prime}} \\
&=\left(Q_{m}\right)_{P^{\prime}}+\left(Q_{m x}\right)_{P^{\prime}} d x+\left(Q_{m y}\right)_{P^{\prime}} d y+\left(Q_{m t}\right)_{P^{\prime}} d t \\
& \quad+\frac{1}{2}\left(Q_{m x x}\right)_{P^{\prime}}(d x)^{2}+\frac{1}{2}\left(Q_{m y y}\right)_{P^{\prime}}(d y)^{2} \\
& \quad+\frac{1}{2}\left(Q_{m t t}\right)_{P^{\prime}}(d t)^{2}+\left(Q_{m x t}\right)_{P^{\prime}}(d x \cdot d t) \\
&+\left(Q_{m y t}\right)_{P^{\prime}}(d y \cdot d t)+\left(Q_{m x y}\right)_{P^{\prime}}(d x \cdot d y) \\
& E_{m}(d x, d y, d t)_{P^{\prime}} \\
&=\left(E_{m}\right)_{P^{\prime}}+\left(E_{m x}\right)_{P^{\prime}} d x+\left(E_{m y}\right)_{P^{\prime}} d y+\left(E_{m t}\right)_{P^{\prime}} d t \\
&+\frac{1}{2}\left(E_{m x x}\right)_{P^{\prime}}(d x)^{2}+\frac{1}{2}\left(E_{m y y}\right)_{P^{\prime}}(d y)^{2} \\
&+ \frac{1}{2}\left(E_{m t t}\right)_{P^{\prime}}(d t)^{2}+\left(E_{m x t}\right)_{P^{\prime}}(d x \cdot d t) \\
&+\left(E_{m y t}\right)_{P^{\prime}}(d y \cdot d t)+\left(E_{m x y}\right)_{P^{\prime}}(d x \cdot d y) \\
& F_{m}(d x, d y, d t)_{P^{\prime}} \\
&=\left(F_{m}\right)_{P^{\prime}}+\left(F_{m x}\right)_{P^{\prime}} d x+\left(F_{m y}\right)_{P^{\prime}} d y+\left(F_{m t}\right)_{P^{\prime}} d t \\
&+\frac{1}{2}\left(F_{m x x}\right)_{P^{\prime}}(d x)^{2}+\frac{1}{2}\left(F_{m y y}\right)_{P^{\prime}}(d y)^{2} \\
&+\frac{1}{2}\left(F_{m t t}\right)_{P^{\prime}}(d t)^{2}+\left(F_{m x t}\right)_{P^{\prime}}(d x \cdot d t) \\
&\left(F_{m y t}\right)_{P^{\prime}}(d y \cdot d t)+\left(F_{m x y}\right)_{P^{\prime}}(d x \cdot d y)
\end{aligned}
$$

Here, $d x=x-x_{P^{\prime}}, d y=y-y_{P^{\prime}}, d t=t-t_{P^{\prime}}$, where $x_{p^{\prime}}$, $y_{p^{\prime}}$ and $t_{p^{\prime}}$ are the position coordinates of point $P^{\prime}$.

Substituting equation (11) into equation (9) gives

$$
\begin{aligned}
& \left(Q_{m t}\right)_{P^{\prime}}=-\left(E_{m x}\right)_{P^{\prime}}-\left(F_{m y}\right)_{P^{\prime}}+\left(S_{m}\right)_{P^{\prime}} \\
& \left(Q_{m x t}\right)_{P^{\prime}}=-\left(E_{m x x}\right)_{P^{\prime}}-\left(F_{m x y}\right)_{P^{\prime}}+\left(S_{m x}\right)_{P^{\prime}} \\
& \left(Q_{m y t}\right)_{P^{\prime}}=-\left(E_{m x y}\right)_{P^{\prime}}-\left(F_{m y y}\right)_{P^{\prime}}+\left(S_{m y}\right)_{P^{\prime}} \\
& \left(Q_{m t t}\right)_{P^{\prime}}=-\left(E_{m x t}\right)_{P^{\prime}}-\left(F_{m y t}\right)_{P^{\prime}}+\left(S_{m t}\right)_{P^{\prime}}
\end{aligned}
$$

The above equations imply that the variables required in computation are $\left(Q_{m}\right)_{P^{\prime}},\left(Q_{m x}\right)_{P^{\prime}}$ and $\left(Q_{m y}\right)_{P^{\prime}}$, because $S_{m}$, $E_{m}$ and $F_{m}$ are the function of $Q_{m}$. Define the $\mathrm{CE}\left(P^{\prime}\right)$ as illustrated in Figure 2(c). $\mathrm{CE}\left(P^{\prime}\right)$ is related to not only $\mathrm{SE}\left(P^{\prime}\right)$ but also the $\mathrm{SE}$ of $\mathrm{SE}(\mathrm{A}), \mathrm{SE}(\mathrm{C}), \mathrm{SE}(\mathrm{E})$ and $\mathrm{SE}(\mathrm{G})$. Note that the values of physical variables on mesh point $A$, $\mathrm{C}$, $\mathrm{E}$ and $\mathrm{G}$ are known. Assume that the integral conservation laws are satisfied in every CE. Integrating equation (10) on the surfaces of $\operatorname{CE}\left(P^{\prime}\right)$ with the aid of equation (12), we find

$$
\begin{aligned}
& \left(Q_{m}\right)_{P^{\prime}}+\frac{1}{24} \frac{\Delta x}{\Delta y}\left(Q_{m x x}\right)_{P^{\prime}}+\frac{1}{24} \frac{\Delta y}{\Delta x}\left(Q_{m y y}\right)_{P^{\prime}}-\frac{\Delta t}{4}\left(S_{m}\right)_{P^{\prime}} \\
& =\frac{1}{4}\left(\bar{Q}+\frac{\Delta t}{\Delta x} \bar{E}+\frac{\Delta t}{\Delta y} \bar{F}+\frac{\Delta t}{4} \bar{S}\right)
\end{aligned}
$$

where

$$
\begin{aligned}
\bar{Q}= & Q_{m}\left(A, \frac{\Delta x}{4}, \frac{\Delta y}{4}, 0\right)+Q_{m}\left(C,-\frac{\Delta x}{4}, \frac{\Delta y}{4}, 0\right) \\
& +Q_{m}\left(E,-\frac{\Delta x}{4},-\frac{\Delta y}{4}, 0\right) \\
& +Q_{m}\left(G, \frac{\Delta x}{4},-\frac{\Delta y}{4}, 0\right) \\
\bar{E}= & E_{m}\left(A, 0, \frac{\Delta y}{4}, \frac{\Delta t}{4}\right)-E_{m}\left(C, 0, \frac{\Delta y}{4}, \frac{\Delta t}{4}\right) \\
& -E_{m}\left(E, 0,-\frac{\Delta y}{4}, \frac{\Delta t}{4}\right) \\
& +E_{m}\left(G, 0,-\frac{\Delta y}{4}, \frac{\Delta t}{4}\right) \\
\bar{F}= & F_{m}\left(A, \frac{\Delta x}{4}, 0, \frac{\Delta t}{4}\right)+F_{m}\left(C,-\frac{\Delta x}{4}, 0, \frac{\Delta t}{4}\right) \\
& -F_{m}\left(E,-\frac{\Delta x}{4}, 0, \frac{\Delta t}{4}\right) \\
& -F_{m}\left(G, \frac{\Delta x}{4}, 0, \frac{\Delta t}{4}\right) \\
\bar{S}= & S_{m}(A)+S_{m}(C)+S_{m}(E)+S_{m}(G)
\end{aligned}
$$

Using the continuity conditions at points $A^{\prime}, C^{\prime}, E^{\prime}$ and $G^{\prime}$, the derivatives of $Q_{m}$ with respect to $x$ and $y$ are obtained

$$
\begin{aligned}
& \left(Q_{x}\right)_{P^{\prime}}=W\left[\left(Q_{x}\right)_{P^{\prime}}^{-},\left(Q_{x}\right)_{P^{\prime}}^{+}, \alpha\right] \\
& \left(Q_{y}\right)_{P^{\prime}}=W\left[\left(Q_{y}\right)_{P^{\prime}}^{-},\left(Q_{y}\right)_{P^{\prime}}^{+}, \alpha\right] \\
& \left(Q_{m x x}\right)_{P^{\prime}}=\frac{1}{\Delta x}\left[\left(Q_{m x}\right)_{D^{\prime}}-\left(Q_{m x}\right)_{H^{\prime}}\right] \\
& \left(Q_{m y y}\right)_{P^{\prime}}=\frac{1}{\Delta y}\left[\left(Q_{m y}\right)_{F^{\prime}}-\left(Q_{m y}\right)_{B^{\prime}}\right] \\
& \left(Q_{m x y}\right)_{P^{\prime}}=\frac{1}{2}\left[\left(\hat{Q}_{m x y}\right)_{P^{\prime}}+\left(\hat{Q}_{m y x}\right)_{P^{\prime}}\right]
\end{aligned}
$$

where 


$$
\begin{aligned}
\left(Q_{m x}\right)_{D^{\prime}}= & {\left[Q_{m x}(C, 0,0, \Delta t / 2)+Q_{m x}(E, 0,0, \Delta t / 2)\right] / 2 } \\
\left(Q_{m x}\right)_{H^{\prime}}= & {\left[Q_{m x}(G, 0,0, \Delta t / 2)+Q_{m x}(A, 0,0, \Delta t / 2)\right] / 2 } \\
\left(Q_{m y}\right)_{F^{\prime}}= & {\left[Q_{m y}(E, 0,0, \Delta t / 2)+Q_{m y}(G, 0,0, \Delta t / 2)\right] / 2 } \\
\left(Q_{m y}\right)_{B^{\prime}}= & {\left[Q_{m y}(A, 0,0, \Delta t / 2)+Q_{m y}(C, 0,0, \Delta t / 2)\right] / 2 } \\
\left(\bar{Q}_{m x y}\right)_{P^{\prime}}= & {\left[Q_{m x}(C, 0,0, \Delta t / 2)-Q_{m x}(A, 0,0, \Delta t / 2)\right.} \\
& +Q_{m x}(E, 0,0, \Delta t / 2) \\
& \left.-Q_{m x}(G, 0,0, \Delta t / 2)\right] /(2 \Delta y) \\
\left(\widehat{Q}_{m y x}\right)_{P^{\prime}}= & {\left[Q_{m y}(C, 0,0, \Delta t / 2)-Q_{m y}(A, 0,0, \Delta t / 2)\right.} \\
& +Q_{m y}(E, 0,0, \Delta t / 2) \\
& \left.-Q_{m y}(G, 0,0, \Delta t / 2)\right] /(2 \Delta x)
\end{aligned}
$$

Here $\left(Q_{x}\right)_{P^{\prime}}^{ \pm},\left(Q_{y}\right)_{P^{\prime}}^{ \pm}$are defined as such

$$
\begin{aligned}
& \left(Q_{m x}\right)_{P^{\prime}}^{-} \\
& =-\frac{1}{\Delta x}\left[Q_{m}\left(A, 0,0, \frac{\Delta t}{2}\right)+Q_{m}\left(G, 0,0, \frac{\Delta t}{2}\right)-2\left(Q_{m}\right)_{P^{\prime}}\right] \\
& \left(Q_{m x}\right)_{P^{\prime}}^{+} \\
& =+\frac{1}{\Delta x}\left[Q_{m}\left(C, 0,0, \frac{\Delta t}{2}\right)+Q_{m}\left(E, 0,0, \frac{\Delta t}{2}\right)-2\left(Q_{m}\right)_{P^{\prime}}\right] \\
& \left(Q_{m y}\right)_{P^{\prime}}^{-} \\
& =-\frac{1}{\Delta y}\left[Q_{m}\left(A, 0,0, \frac{\Delta t}{2}\right)+Q_{m}\left(C, 0,0, \frac{\Delta t}{2}\right)-2\left(Q_{m}\right)_{P^{\prime}}\right] \\
& \left(Q_{m y}\right)_{P^{\prime}}^{+} \\
& =+\frac{1}{\Delta y}\left[Q_{m}\left(E, 0,0, \frac{\Delta t}{2}\right)+Q_{m}\left(G, 0,0, \frac{\Delta t}{2}\right)-2\left(Q_{m}\right)_{P^{\prime}}\right]
\end{aligned}
$$

The weighted equation is $W\left[x_{+}, x_{-}, \beta\right]=\frac{\left|x_{+}\right|^{\beta} x_{-}+\left|x_{-}\right|^{\beta} x_{+}}{\left|x_{-}\right|^{\beta}+\left|x_{-}\right|^{\beta}}$, and $\beta$ is a constant ( $\beta=2$ in this study) (Chang, 1995).

It should be noted that $\left(Q_{m}\right)_{P^{\prime}}$ can not be obtained explicitly from equation (13) due to the source term $\left(S_{m}\right)_{P^{\prime}}$. As $\left(S_{m}\right)_{P^{\prime}}$ is a function of $\left(Q_{m}\right)_{P^{\prime}}$, a local Newton iterative procedure is usually needed to determine $\left(Q_{m}\right)_{P^{\prime}}$.. In the present work, to avoid the iterative procedure and save computation time, $\left(S_{m}\right)_{P^{\prime}}$ is replaced by their linear prediction of current time in equation (13), i.e.,

$$
\begin{aligned}
& \left(Q_{m}\right)_{P^{\prime}}+\frac{1}{24} \frac{\Delta x}{\Delta y}\left(Q_{m x x}\right)_{P^{\prime}}+\frac{1}{24} \frac{\Delta y}{\Delta x}\left(Q_{m y y}\right)_{P^{\prime}} \\
& \left.=\frac{1}{4}\left(\bar{Q}+\frac{\Delta t}{\Delta x} \bar{E}+\frac{\Delta t}{\Delta y} \bar{F}\right)+\frac{\Delta t}{8} \bar{S}\right) \\
& \tilde{S}=\bar{S}+\frac{\Delta t}{4} \bar{S}_{t}
\end{aligned}
$$

where $\bar{S}_{t}$ is the time derivative of $\bar{S} .\left(Q_{m}\right)_{P^{\prime}}$ can be directly solved without any iteration, since the time derivative of $\left(S_{m}\right)_{A},\left(S_{m}\right)_{E},\left(S_{m}\right)_{C}$ and $\left(S_{m}\right)_{G}$ are all known at current time.

\subsection{Pretreatment procedure}

The concept of the pretreatment method is proposed, which converts the elliptic-parabolic equations to the hyperbolic equations, which are accurately solved by the CE/SE method. Based on the artificial compressibility method (Chorin, 1967) coupling velocity and pressure, equation (1) can be rewritten in form of

$$
\frac{\partial P}{\partial \tau}+C^{2} \rho\left(\frac{\partial u}{\partial x}+\frac{\partial v}{\partial y}\right)=0
$$

where $\tau$ is the virtual time, and $C^{2}$ is the artificial compressibility coefficient effecting the numerical stability. Substituting the virtual time derivative of velocities into equation (9), we obtain

$$
\frac{\partial U}{\partial \tau}+\frac{\partial Q}{\partial t}+\frac{\partial E(Q)}{\partial x}+\frac{\partial F(Q)}{\partial y}=S(Q)
$$

Here $U=\left[\begin{array}{c}\frac{1}{C^{2}} P \\ u \\ v\end{array}\right]$, for $\tau \rightarrow \infty$, equation (20) is consistent to equation (9). Applying the pressure splitting method, equation (20) takes the form

$$
\frac{\partial U_{v}}{\partial \tau}+\frac{\partial Q_{v}}{\partial t}+R_{v}\left(Q_{v}\right)+\frac{1}{\rho} \nabla P=0
$$

with

$$
\begin{aligned}
& R_{v}\left(Q_{v}\right)=\left(\frac{\partial E_{v}\left(Q_{v}\right)}{\partial x}+\frac{\partial F_{v}\left(Q_{v}\right)}{\partial y}-S_{v}\left(Q_{v}\right)\right), \\
& R_{v}\left(Q_{v}\right)=\left(\frac{\partial E_{v}\left(Q_{v}\right)}{\partial x}+\frac{\partial F_{v}\left(Q_{v}\right)}{\partial y}-S_{v}\left(Q_{v}\right)\right), \\
& Q_{v}=U_{v}=\left[\begin{array}{l}
u \\
v
\end{array}\right], \quad E_{v}=\left[\begin{array}{l}
u^{2}-\frac{\tau_{x x}}{\rho \operatorname{Re}} \\
u v-\frac{\tau_{y x}}{\rho \operatorname{Re}}
\end{array}\right], F_{v}=\left[\begin{array}{c}
u v-\frac{\tau_{x y}}{\rho \operatorname{Re}} \\
v^{2}-\frac{\tau_{y y}}{\rho \operatorname{Re}}
\end{array}\right], \\
& S_{v}=\left[\begin{array}{l}
\frac{g_{x}}{F r}+\frac{\kappa(\varphi) \delta_{e}(\varphi)}{\bar{\rho} W e} \frac{\partial \varphi}{\partial x} \\
\frac{g_{y}}{F r}+\frac{\kappa(\varphi) \delta_{e}(\varphi)}{\bar{\rho} W e} \frac{\partial \varphi}{\partial y}
\end{array}\right]
\end{aligned}
$$


Figure 3 Streamlines on (a) $50 \times 50$ grid, (b) $100 \times 100$ grid, and (c) $128 \times 128$ grid for $\operatorname{Re}=1,000$

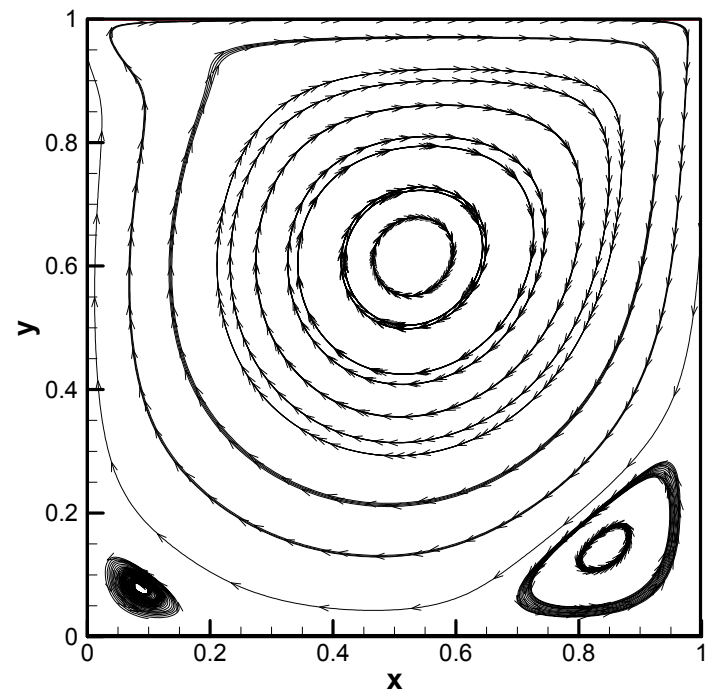

(a)

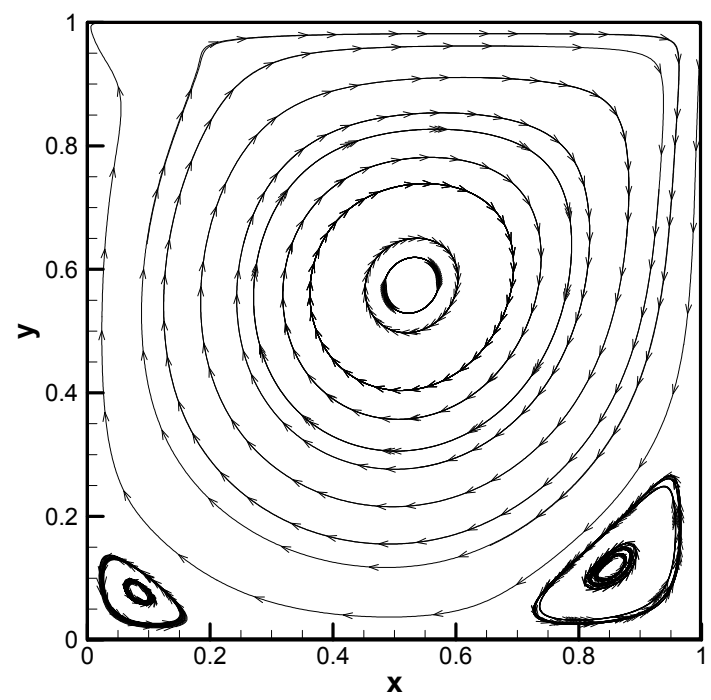

(b)

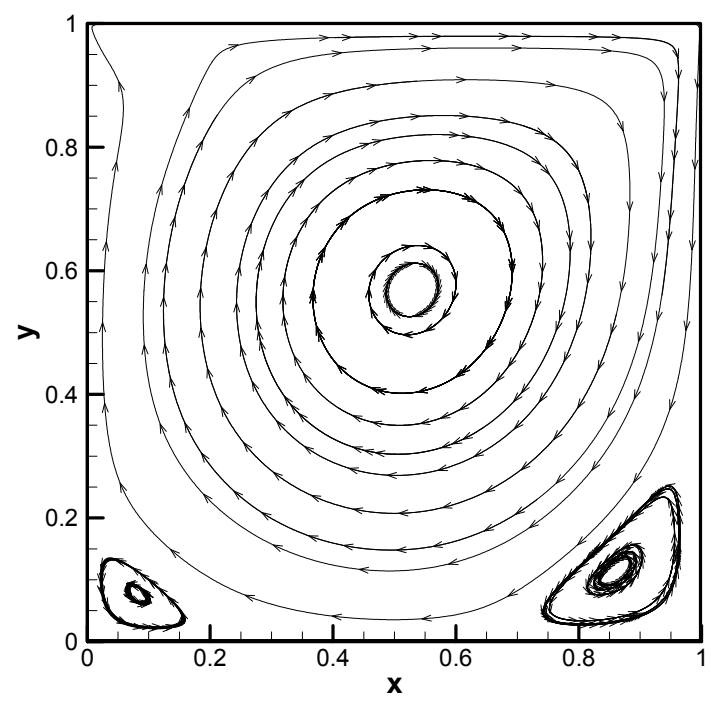

(c)
Figure 4 Velocity profiles through the cavity centre for $\mathrm{Re}=100$ and $\mathrm{Re}=1,000$, (a) $u$ component along the vertical line through the cavity centre (grid of $128 \times 128$ ) (b) $v$ component along the horizontal line through the cavity centre (grid of $100 \times 100$ ), lines denote $\mathrm{CE} / \mathrm{SE}$ solutions and symbols present benchmark solutions in Ghia et al. (1982)

(c) $v$ component at the cavity's horizontal centreline on $50 \times 50$ grid and on $100 \times 100$ grid for $\mathrm{Re}=1,000$

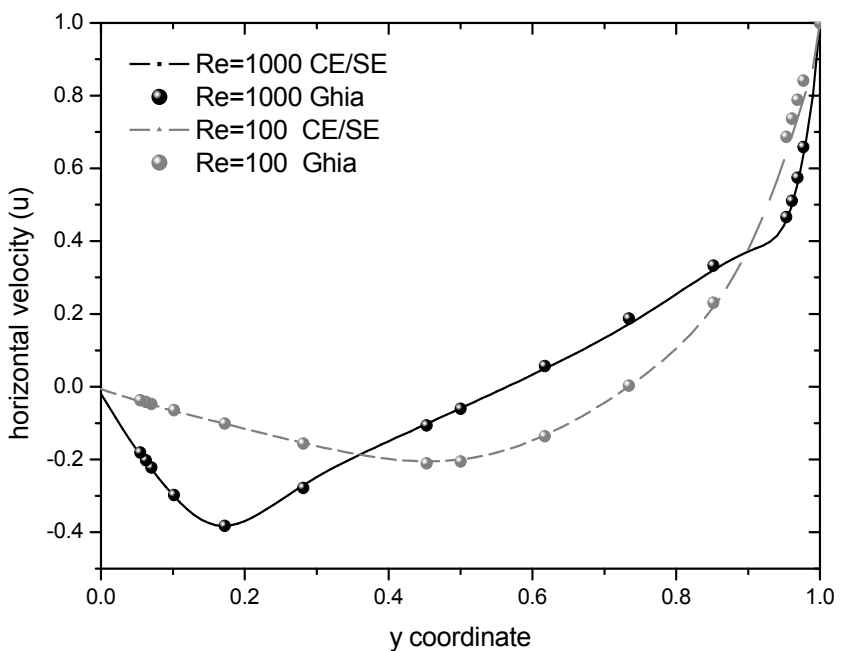

(a)

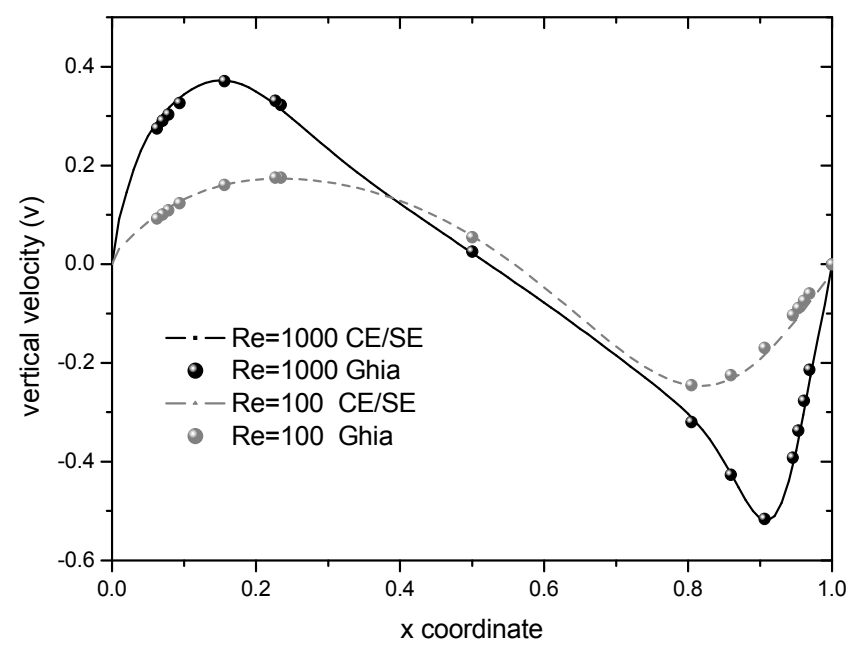

(b)

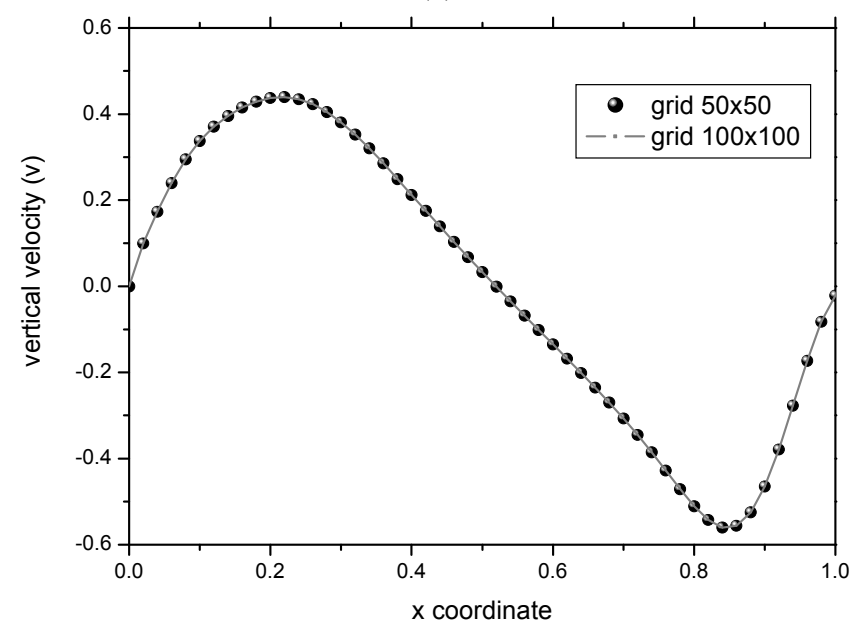

(c) 
Using the time operator splitting method to split pressure item in momentum equation (21), we obtain

$$
\begin{aligned}
& \frac{Q_{v}^{n+\frac{1}{2}}-f\left(Q_{v}^{n}\right)}{\Delta t}+R_{v}^{n}\left(Q_{v}^{n}\right)=0 \\
& \frac{\partial U}{\partial \tau}+\frac{Q_{v}^{n+1}-Q_{v}^{n+\frac{1}{2}}}{\Delta t}+\frac{1}{\rho} \nabla P=0
\end{aligned}
$$

We interpret equation (22) as yielding an intermediate value of $Q_{v}^{n}$, denoted by $Q_{v}^{n+\frac{1}{2}}$ at time $n$. Here, the superscript $n$ indicates the time step. $F\left(Q_{v}^{n}\right)$ is the value of $Q_{v}^{n}$ at time step $n$. Equation (22) can be simulated by CE/SE method to obtain $Q_{v}^{n+\frac{1}{2}}$. By means of time-marching solutions and internal iteration method, equations (19) and (23) yield

$$
\begin{aligned}
& \frac{P^{m+1}-f\left(P^{m}\right)}{\Delta \tau}+C^{2}\left(\frac{\partial u}{\partial x}+\frac{\partial v}{\partial y}\right)^{m}=0 \\
& \frac{U^{m+1}-U^{m}}{\Delta \tau}+\frac{Q^{m+1}-Q^{n+\frac{1}{2}}}{\Delta t}+\frac{1}{\rho} \nabla P^{m+1}=0
\end{aligned}
$$

where $m$ is the iteration step. Assume that we have already carried out $m$ iteration. Then, for the $m+1$ iteration, by substituting $Q_{v}^{n+\frac{1}{2}}$ into equation (24) and using $\mathrm{CE} / \mathrm{SE}$ method, $P^{m+1}$ can be calculated at iteration step $m+1$. Substituting $P^{m+1}$ into equation (25), $U^{m+1}$ is obtained. The above procedure is repeated for a number of iteration, convergence is achieved when $U^{m+1}-U^{m}$ become less than a prescribed value $\left(10^{-6}\right)$ at all grid points. After convergence, $U^{n+1}$ can be obtained at time step $n+1$. These governing equations are discretised on staggered orthogonal grid in order to eliminate the possibility of a checkerboard pressure pattern.

\section{Numerical validation}

In the following, the improved CE/SE scheme performance is examined on five test cases. The first case is the steady-state single-phase flow calculation. The cases 2 to 5 are multiphase flow problems.

\subsection{Driven cavity flow}

The square lid-driven cavity (Ghia et al. 1982) is a well-known benchmark problem for numerical methods, where the flow is driven by the friction force, and the viscosity mainly controls the flow. Here, three sets of grid $(50 \times 50,100 \times 100$ and $128 \times 128)$ are used, and $\operatorname{Re}=1,000$ and $\operatorname{Re}=100$ are simulated by using the improved CE/SE scheme. Figures 3(a) to 3(c) shows the streamline. There is a primary vortex inside the cavity, and two smaller reverse-rotating vortices occur at both corners of the bottom wall. This flow structure is similar as that in Ghia's result (1982). Even on a coarse grid resolution $(50 \times 50)$. The $\mathrm{CE} / \mathrm{SE}$ method produces results that agree well with the reference result of Ghia et al. on the grid of $128 \times 128$. Figure $4($ a) shows the $u$ velocity distribution (grid of $128 \times 128$ ) along the vertical centreline and is compared with Ghia's data. Figure 4(b) depicts the $v$ velocity distribution (grid of $100 \times 100$ ) along the horizontal centreline. It can be seen that the result of the finer mesh $(128 \times 128$ and $100 \times 100)$ matches very well with Ghia's. Note that a $128 \times 128$ mesh was used in Ghia's work. In addition, Figure 4(c) depicts the calculated $v$ velocity profiles at the cavity's horizontal centreline for grid sensitivity test. Notably, due to the CE/SE high accuracy, the $\mathrm{CE} / \mathrm{SE}$ results on $50 \times 50$ grid are as good as the solution on $100 \times 100$ grid.

\subsection{Dam break}

For further validation of the improved CE/SE scheme coupled with HPLS method, the dam break problem (Martin and Moyce, 1952) is numerically calculated. A $81 \times 41$ uniform Cartesian grid is applied with an initial water column height to width ratio of 2 . Densities of water and air are $1,000 \mathrm{~kg} / \mathrm{m}^{3}, 1 \mathrm{~kg} / \mathrm{m}^{3}$, respectively. Viscosities of water and air are $0.001 \mathrm{~kg} /(\mathrm{ms}), 0.00001 \mathrm{~kg} /(\mathrm{ms})$, respectively. The calculated domain is $0.285 \mathrm{~m} \times 0.0675 \mathrm{~m}$. At the outlet boundary, the Neumann boundary condition is set for velocities. At all other boundaries, slip wall boundary conditions are applied. Figure 5(a) shows the phase interface profiles between time of $0.0 \mathrm{~s}$ and time of $0.24 \mathrm{~s}$ with time interval $0.08 \mathrm{~s}$. The water surface evolves in a smooth shape and no oscillation occurs at the interface near the solid wall. Figure 5(b) depicts the history of the water front marching along the ground surface $(y=0)$. The numerical results are in well agreement with the experimental data (Martin and Moyce, 1952).

\subsection{Drop falling flows}

$\mathrm{Ni}$ et al (2006) studied the effects of wall repulsion, inertia, and deformation on the motions of falling droplets, and considered the interaction of two and three droplets in a channel. Here, the improved CE/SE scheme and HPLS method are used to numerically investigate these physical processes of some cases. The computational grid $100 \times 200$ is used. The droplet motions calculated by the improved $\mathrm{CE} / \mathrm{SE}$ are demonstrated in Figure 6. Figures 6(a) and 6(b) shows the effects of the wall on the deformation and rotation of the droplet. Figure 6(c) illustrates the effect of the two droplets repulsion process. Figure 6(d) depicts the repulsion and droplets coalesce, indicating the effect of inter-droplet and wall repulsion. From Figures 6(a) to 6(d) we can find that the improved $\mathrm{CE} / \mathrm{SE}$ scheme performs well agreement with the results by projection method coupling with the level set method (Ni et al., 2006). 


\subsection{Viscous Rayleigh-Taylor instability}

Rayleigh-Taylor instability problem is commonly used as the benchmark problem to investigate the effects of fluid density differences and viscosity ratios on the instability of immiscible flows. To initiate the instability at the fluid-fluid interface, the initially flat front was perturbed by a sinusoidal deformation (Guermond and Quartapelle, 2000.). Here, we considered displacement of a less dense fluid by a denser fluid. We consider a two-dimensional Rayleigh-Taylor instability problem in a domain of size
$[1 \times 4]$. A computational grid $100 \times 400$ is used. The ratio of viscosity and density are 1 and 3, respectively, for $\operatorname{Re}=1,000$. The slip boundary conditions are applied to the left and right sides, while non-slip boundaries for the top and down sides. Figure 7 shows the CE/SE calculated dynamics of the interface at times 1, 1.75, 2 and 2.5. As time increases, the mass interface transforms from a single smooth finger to a highly unstable fragmented dendritic. Results by the improved CE/SE scheme well satisfy with results by Guermond and Quartapelle (2000).

Figure 5 (a) Evolution of the free interface and (b) history of water front location at $y=0$ from time $=0.0 \mathrm{~s}$ to time $=0.24 \mathrm{~s}$ with time interval $0.08 \mathrm{~s}$

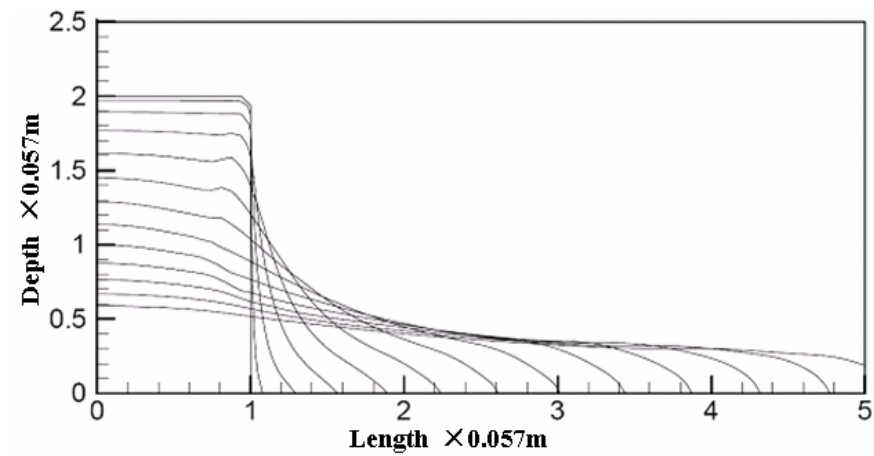

(a)

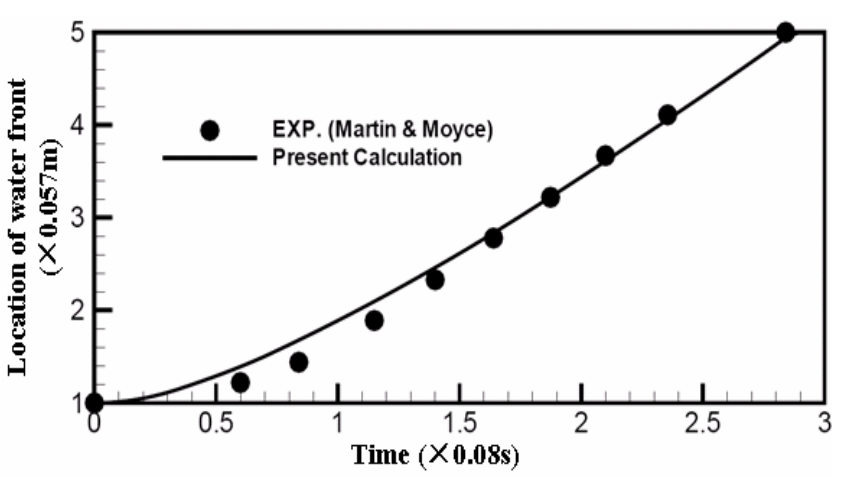

(b)

Figure 6 Droplet falling, (a) near the wall with initial position $(1.5,16.5)$ and (b) in the central region with initial position at (4.5, 16.5) for $\operatorname{Re}=100, W e=50$, viscosity ratio of 1.125 , density ratio of 50; Falling two-droplet flows near the wall with initial positions $(1.5,16.5)$ and $(7.5,16.5)$ for $\mathrm{Re}=100$ and $W e=50(\mathrm{c})$ at viscosity ratio of 1.125 and density ratio of 50 and (d) viscosity ratio of 50 and density ratio of 1.125

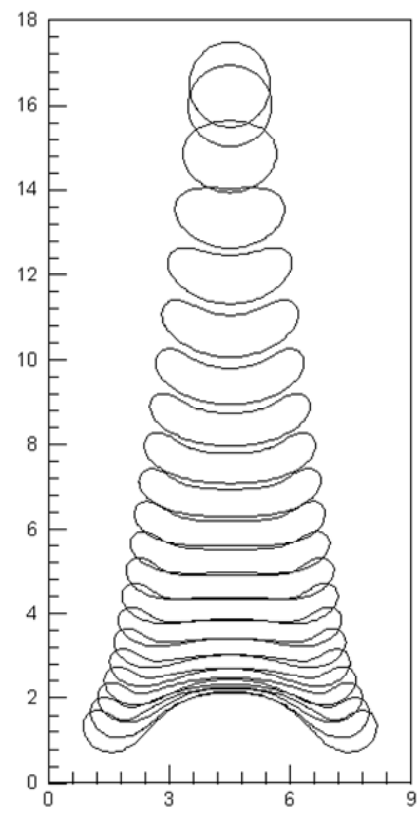

(a)

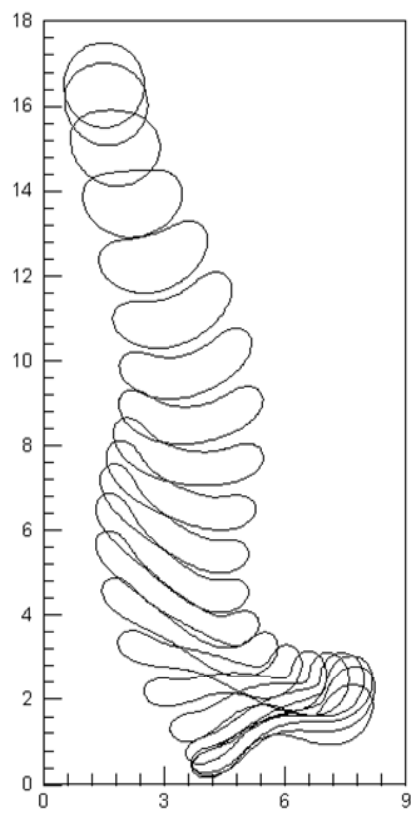

(b)

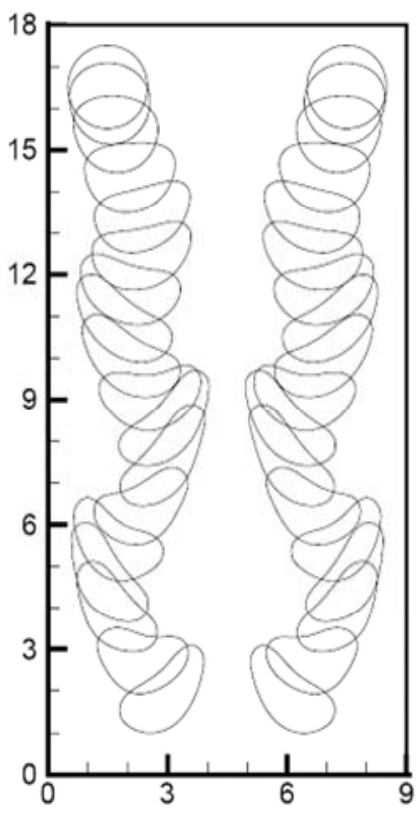

(c)

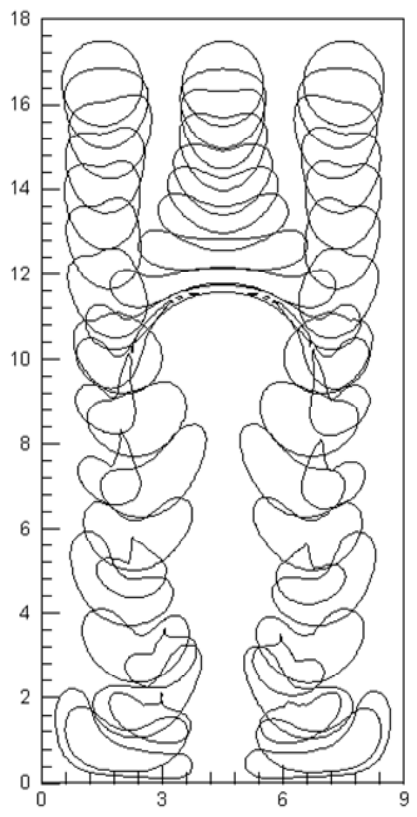

(d)

Note: The viscosity and density ratio denote the ratio of viscosity and density of the drop to that of the background fluid, respectively. 
Figure 7 Dynamics of interface calculated by the improved CE/SE scheme at times (a) $t=1$, (b) $t=1.75$, (c) $t=2$, and (d) $t=2.5$

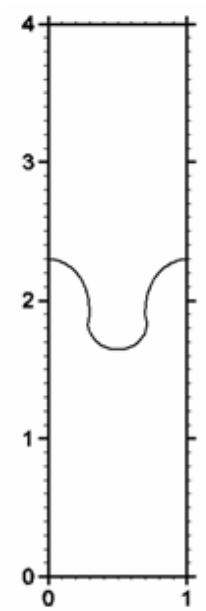

(a)

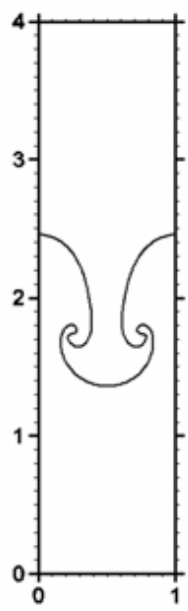

(b)

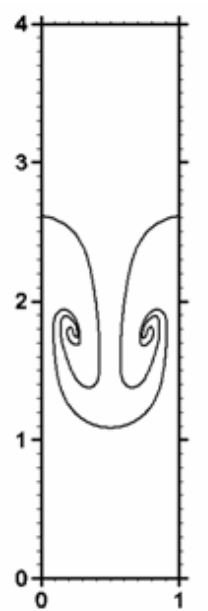

(c)

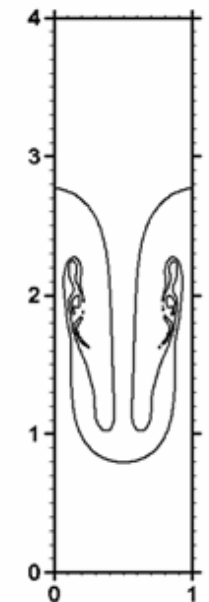

(d)

\subsection{Water and kerosene interaction in T-shaped tube}

Dong et al (2008) set up an experiment, in which two-phase flow problem in a T-shaped micro-fracture was considered. The width and depth of the T-shaped micro-fracture are $6 \mathrm{~mm}, 3 \mathrm{~mm}$, respectively. The size of $L$ is $6 \mathrm{~mm}$. For detailed information about the experimental instruments and the experimental process, readers refer to the lecture (Dong et al., 2008). The motion of the mass interface and the mixture as well as separation process are observed by CCD-Scanning system. This process is also numerically simulated by the improved CE/SE scheme coupled with HPLS method, by simplifying the 3D (three dimensional) problem into the $2 \mathrm{D}$ problem as shown in Figure 8(a). The mesh grids are $100 \times 200$ and the time interval is $0.0005 \mathrm{~s}$. The surface force between the oil, water and solid wall is taken into account. The wall boundary condition is introduced. The left and right boundaries are subject to the inlet condition and the outlet boundary is imposed on the bottom. The contact angle boundary is assigned to the interface between the wall and fluids by level-set function. The parameters used are summarised in Tables 1 and 2 . When the flow reaches the stable state, the experimental data and the improved $\mathrm{CE} / \mathrm{SE}$ calculated results are demonstrated in Figures 8(b) to 8(c). The interface between water and kerosene is clear. The improved CE/SE scheme accurately captures the interface and the separation process. It can be seen that the numerical results by the improved CE/SE scheme coupled with HPLS method match the experimental data well.

\section{Conclusions}

In this paper, the improved $\mathrm{CE} / \mathrm{SE}$ scheme is proposed for incompressible multiphase flows. This scheme is based on the rectangular mesh and can be constructed easily. The fluid-fluid interfaces are traced by the high-resolution HPLS technique. The main advantages of the improved $\mathrm{CE} / \mathrm{SE}$ scheme are clear in physical concept, accurate for capturing fluid-fluid interface, easy to be extended to threedimensional situations, since the CE/SE scheme and the HPLS technique both easy to be extended to three dimensions. The improved $\mathrm{CE} / \mathrm{SE}$ scheme exhibits an excellent performance in all five test problems examined in the preceding sections. The numerical results show that the improved CE/SE scheme can accurately simulate incompressible multiphase flows. Thus, the improved $\mathrm{CE} / \mathrm{SE}$ scheme can be applied to incompressible multiphase flow simulations for academic explore and engineering widely. The explicit treatment and algorithmic simplicity of the improved $\mathrm{CE} / \mathrm{SE}$ provide additional advantages for massively parallel computations. The authors note that the code of the improved CE/SE scheme is open to the public.

Figure 8 Concept model and experimental (Dong et al., 2008) data and the improved CE/SE calculated results for $\operatorname{Re}=83.1, W e=0.514$ (i), $\operatorname{Re}=5.54$, $W e=0.00228$ (ii) and $\operatorname{Re}=55.4, W e=0.228$ (iii), respectively, (a) conceptual model (b) experiment (c) $\mathrm{CE} / \mathrm{SE}$ calculated (see online version for colours)

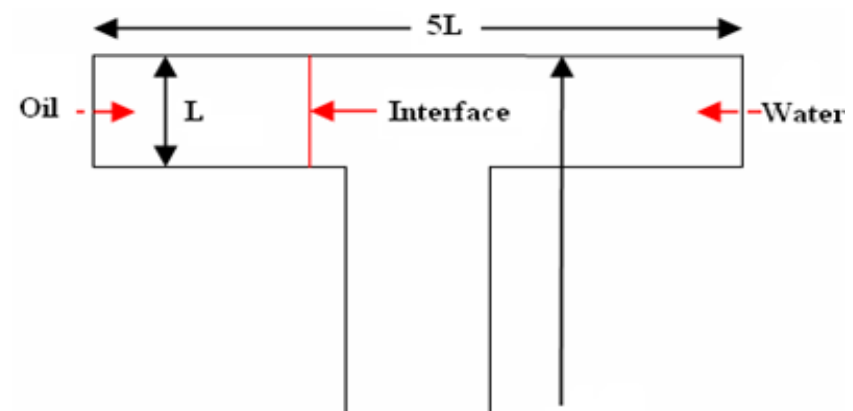

10L

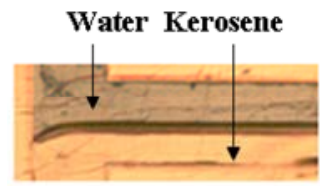

Water Kerosene
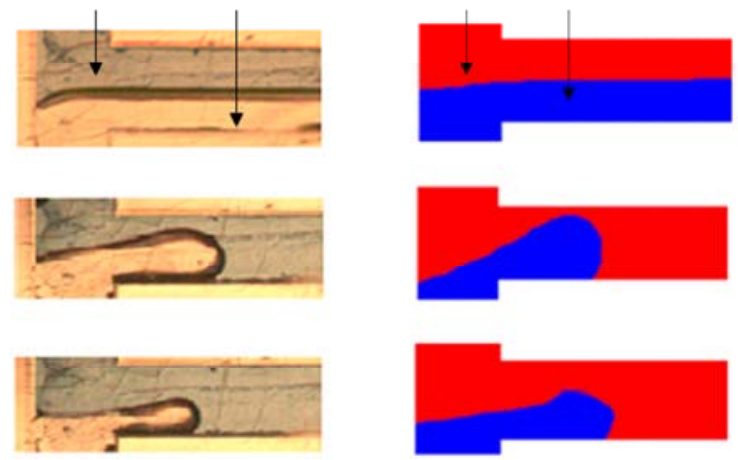

(c) 
Table 1 Property of kerosene and water used in this model

\begin{tabular}{lccc}
\hline Fluid & $\begin{array}{c}\text { Density } \\
\left(\mathrm{kg} / \mathrm{m}^{3}\right)\end{array}$ & $\begin{array}{c}\text { Viscosity } \\
(\text { Pas })\end{array}$ & $\begin{array}{c}\text { Surface force } \\
\left(\mathrm{Nm}^{-1}\right)\end{array}$ \\
\hline Kerosene & 780 & 0.000115 & 0.045 \\
Water & 998.2 & 0.001 & \\
\hline
\end{tabular}

Table 2 Calculated parameters for three cases

\begin{tabular}{lcccc}
\hline Inlet velocity $(\mathrm{m} / \mathrm{s})$ & Re & We & Case \\
\hline $0.2778^{\mathrm{K}}$ & $-0.2778^{\mathrm{W}}$ & 83.1 & 0.514 & 1 \\
$0.0185^{\mathrm{K}}$ & $-0.00093^{\mathrm{W}}$ & 5.54 & 0.00228 & 2 \\
$0.185^{\mathrm{K}}$ & $-0.00926^{\mathrm{W}}$ & 55.4 & 0.228 & 3 \\
\hline
\end{tabular}

Notes: Minus '-' denotes the flow direction. Index of K and $\mathrm{W}$ presents kerosene and water, respectively.

\section{Acknowledgements}

This research is supported by the National Basic Research Program of China, Fundamental research of carbon dioxide emission reduction, geological storage and utilization (FCGU) 2011CB707300, Wenchuan Fault Scientific Drilling Project of China (WFSD), and the Institute of Crustal Dynamics, CEA under Grant No. ZDJ2010-26.

\section{References}

Brackbill, J.U., Kothe, D.B. and Zemach, C. (1992) 'A continuum method for modeling surface tension', Journal of Computational Physics, Vol. 100, No. 2, pp.335-354.

Chang, S.C. (1995) 'The method of space-time conservation element and solution element-a new approach for solving the Navier-Stokes and Euler equations', Journal of Computational Physics, Vol. 119, No. 2, pp.295-324.

Chang, S.C., Wang, X.Y. and Chow, C.Y. (1999) 'The space-time conservation element and solution element method: a new high-resolution and genuinely multidimensional paradigm for solving conservation laws', Journal of Computational Physics, Vol. 156, No. 1, pp.89-136.

Chorin, A.J. (1967) 'A numerical method for solving incompressible viscous flow problems', Journal of Computational Physics, Vol. 2, No. 12 (recent edition, 1997: Vol. 135, pp.118-125).

Dong, H.F., Zhang, D.L. and Zhao, Y.C. (2008) 'Numerical simulation of immiscible two-phase flow in T-shaped micro-tube', Chinese Journal of Chemical Engineering, Vol. 59, No. 8, pp.1950-1957.

Enright, D., Fedkiw, R. and Ferziger, J. (2002) 'A hybrid particle level set method for improved interfaces capturing', Journal of Computational Physics, Vol. 176, No. 1, pp.205-227.

Ghia, U., Ghia, K.N. and Shin, C.T. (1982) 'High-resolutions for incompressible flow using the Navier-Stokes equations and a multigrid method', Journal of Computational Physics, Vol. 48, No. 3, pp.387-411.

Guermond, G.L. and Quartapelle, L. (2000) 'A projection FEM for variable density compressible flows', Journal of Computational Physics, Vol. 165, No. 1, pp.167-188.
Guo, Y.H., Hsu, A.T., Wu, J., Yang, Z.G. and Oyediran, A. (2004) 'Extension of CE/SE method to 2D viscous flows', Computers \& Fluids, Vol. 33, No. 10, pp.1349-1361.

Harvie, D.J.E., Cooper-White, J.J. and Davidson, M. (2008) 'Deformation of a viscoelastic droplet passing through a microfluidic contraction', Journal of Non-Newtonian Fluid Mechanics, Vol. 155, No. 3, pp.67-79.

Martin, J.C. and Moyce, W.J. (1952) 'An experimental study of the collapse of liquid columns on a rigid horizontal plane', Philosophical Transactions of the Royal Society of London Series A-Mathematical Physical and Engineering Sciences, Vol. 244, No. 882, pp.312-324.

Ni, M.J., Komori, S. and Morley, N.B. (2006) 'Direct simulation of falling droplet in a closed channel', International Journal of Heat and Mass Transfer, Vol. 49, Nos. 3-4, pp.366-376.

Nourgaliev, R.R., Dinh, T.N. and Theofanous, T.G. (2004) 'A pseudocompressibility method for the numerical simulation of incompressible multifluid flows', International Journal of Multiphase Flow, Vol. 30, No. 12, pp.901-937.

Nourgaliev, R.R., Dinh, T.N., Theofanous, T.G. and Joseph, D. (2003) 'The Lattice Boltzmann equation method: theoretical interpretation, numerics, and implications', International Journal of Multiphase Flow, Vol. 29, No. 1, pp.117-169.

Osher, S. and Fedkiw, R.P. (2001) 'Level set methods: an overview and some recent results', Journal of Computational Physics, Vol. 169, No. 2, pp.463-502.

Savino, R., Paterna, D. and Lappa, M. (2003) 'Marangoni flotation of liquid droplets', Journal of Fluid Mechanics, Vol. 479, pp.307-326.

Shin, S., Yoon, I. and Juric, D. (2011) 'The local front reconstruction method for direct simulation of two- and three-dimensional multiphase flows', Journal of Computational Physics, Vol. 230, No. 17, pp.6605-6646.

Sibillo, V., Pasquariello, G., Simeone, M., Cristini, V. and Guido, S. (2006) 'Drop deformation in microconfined shear flow', Physical Review Letter, Vol. 97, No. 5, pp.055402(1)-055402(4).

Sussman, M., Smereka, P. and Osher, S. (1994) 'A level set approach for computing solutions to incompressible two-phase flow', Journal of Computational Physics, Vol. 114, No. 1, pp.146-159.

Wang, J.T., Liu, K.X. and Zhang, D.L. (2009) 'An improved $\mathrm{CE} / \mathrm{SE}$ scheme for multi-material elastic-plastic flows and its applications', Computers and Fluids, Vol. 38, No. 3, pp.544-551.

Wang, G., Zhu, H., Zhang, D.L. and Liu, K.X. (2011) 'An improved CE/SE scheme and its application to dilute gas-particle flows', Computer Physics Communications, Vol. 182, No. 1, pp.1589-1601.

Zhang, Z.C., Yu, S.T.J. and Chang, S.C. (2002) 'A space-time conservation element and solution element method for solving the two- and three-dimensional unsteady Euler equations using quadrilateral and hexahedral meshes', Journal of Computational Physics, Vol. 175, No. 1, pp.168-199.

Zhang, M., Yu, S.T. John, Lin, S.C. Henry, Chang, S.C. and Blankson, I. (2006) 'Solving the MHD equations by the space-time conservation element and solution element method', Journal of Computational Physics, Vol. 214, No. 2, pp.599-617. 